IJASC 13-2-2

\title{
Real Examples based Natural Phenomena Synthesis
}

\author{
HyangA An ${ }^{1}$, Yong-Ho Seo², Jinho Park ${ }^{1, \dagger}$ \\ ${ }^{1}$ Global School of Media, Soongsil University, Seoul, South Korea \\ ${ }^{2}$ Dept. of Intelligent Robot Engineering, Mokwon University, Daejeon, South Korea
}

\begin{abstract}
Current physics-based simulation is an important tool in the fluid animation. However some problems require a new change to current research trends which depend only on the simulation. The ultimate goal of this project is to obtain information of flow example, analyze an example through machine learning and the novel fluid animation reconfigure without physical simulation.
\end{abstract}

Key words : Example-based, Physical simulation, Computational efficiency, Vector potential, Tensor

\section{INTRODUCTION}

\subsection{Motivations}

Current physics-based simulation is an important tool in the fluid animation, but the two problems listed below require a new change to current research trends which depend only on the simulation. First, a physics-based method is inefficient in economic terms. Fluid scene used in movies and advertising requires realism level of actual image.

To do this, the huge computation time and computing resources are required. The scene which pours water into a cup for about 10 seconds requires a day or more simulation time. Various studies [6,11] are conducted to calculate efficiency. But variables which are proportional to the resolution of the simulation are woven by the global calculation. There are obvious limitations in efficiency improvements. Second, the current physics-based studies are limitations of representation the subject. Smoke, water, fire, and many of the fluids had the development which makes it incredibly similar to the actual image in terms of the quality of a scene. But at the same time dealing with two or more fluid multi-phase flow [7], such as cigarette smoke, fine fluid diffusion, and other physical objects and interactions [3] and is still considered to be a challenging

Manuscript received: Sept. 15, 2012 / revised : Nov. 20, 2013

Corresponding Author: c2alpha@ssu.ac.kr

Tel: +82-2-828-7040

Global School of Media, Soongsil University problem. Even in the field of computational fluid dynamics, it is true that above problem's physical solutions are on the level of toddlers.

The subject of this study, based on the example fluid animation, is little research that is closely related. Example-based research is an active field of character animation. Various studies [10, 8] using motion capture attracted the attention of many researchers in the last dozen years and in recent years, articulated body dynamics by applying the example of the behavior have been attempted to control character [17]. To reduce the computational load for realistic rendering, studies using examples had been carried out [15] and for the effective deformation calculation, there were studies which analyze simulation in the direction of a particular force in preprocessing step [1]. Example-based idea is spread to fluid simulation, study is carried out that the smoke example which is simulated is simplify the simulation process through data analysis [16]. However, grid resolution to possibly perform real-time is not large enough, as well as, other than smoke such as water it has limitation to extend to fluid.

\subsection{Prospect in the field of fluid animation}

Unlike the main goal that had high-quality output and efficiency improvements fluid results, in the future fluid animation fields are expected to receive attention following the research of three areas. First, in the field of fluid, convergence studies which combine example based ideas will be tried. For the computational efficiency and expand 
the representation of the area, it will be developed from actual image or fluid example consist of simulation result to new animation reconstruction area. Second, the fluid control problems which reflect the diverse needs of users will attract more attention. Like other areas of graphics, fluid field is also advanced form of research that produces results which meet the designer's needs. A high level of control such as specific features added in detail, the appearance of the flow field constrained in a specific time will be carried out by research. Third, parallel computing research is expected to be more prevalent. The methodology [9] which are performed universal calculations on the GPU as theme of the research called GPGPU(General-Purpose computation on Graphics Processing Units) is already spread as CUDA, OpenCL structure. A simulation algorithm which is only consist of region calculation is already performed by GPU and in the future, studies which are even global calculation performed by GPU will be spotlight.

\section{OBJECTIVE}

The ultimate goal of this project is to obtain information of flow example, analyze an example through machine learning and the new fluid animation reconfigure without physical simulation. Fluid data as an example use all data which are video images captured actual fluid and the data which is calculated by captured actual fluid[5] and physics simulation. Depending on user's needs, fluid style selection, the source position, guiding path changing, and the reconstruction of a high-quality video as the same level of example seems more than the ability to perform real-time at 30 frames per second.

\section{OUR METHOD}

As machine learning target, the vector potential [2] for the example velocity field is used. Learning the example velocity field is basic approach. But it can be failed to extract meaningful result. The conservation of mass about incompressible flow is equivalent to $\nabla \cdot \boldsymbol{u}=0$. But if learning only velocity field, it is hard to see reconstructed velocity field is satisfied this condition. After a given velocity field $u$ to the $\boldsymbol{u}=\nabla \times \boldsymbol{p}$ and obtain numerically the vector potential $p$. If this is the object of study, reconstructed velocity field by arbitrary vector field $q$ to the properties of $\nabla \cdot(\nabla \times \boldsymbol{q})=0$ will be always satisfied incompressible fluid field condition.

To overcome the difficulty of high-level examples process, it is attempted to tensor based dimension reduction.
The flow data is proportional to resolution of the sample. So in the case of three-dimensional has generally more millions dimensions. It is common idea in machine learning that if learning these high-level data simply, it is difficult to produce effective result. The flow data can be represented as a multi-dimensional tensor. Therefore it is more effective to apply a tensor-based technology than to apply vector-based dimension reduction such as Principal component analysis.

It is modeling the learning input and output based on the example of time correlation. The decisive viewpoint which is the status change of next time depends only on the current state is the basic principle of physics-based simulation. The model which is approximated from simulation example is Navier-Strokes method so the learning algorithm is tried which is regarded the current frame and the next frame of physics values as features. From the point of machine learning, deterministic perspective can be modeled using the Markov property. Assuming the dimension of derived physical value is properly reduced, the following conditions only depend on current state of feature according to Marko property. That is, Probability which transferred the given current state to the next state is defined as $\mathcal{P}\left(s_{t+1} \mid s_{t}, \alpha\right)$. The next state $s_{t+1}$ and the current state $s_{t}$ became the derived physics values from the example, factors that contribute to the prior state are separately extracted and suppose as $\alpha$, it can learn given example through optimization method of machine learning, and then the next state fluid which is about arbitrary input state can be stochastic selected based on Markov properties.

\section{ACKNOWLEDGMENT}

This work was supported by the National Research Foundation of Korea(NRF) grant funded by the Korean Government (NRF-2012R1A1A2006094).

\section{REFERENCES}

[1] J. Barbič and D. James, "Real-Time subspace integration for St. Venant-Kirchhoff deformable models", ACM Transactions on Graphics (TOG), v.24 n.3, July 2005

[2] R. Bridson, J. Houriham, and M. Nordenstam, "Curl-noise procedural fluid flow", ACM Transactions on Graphics(TOG), vol 26, issue 3, July 2007.

[3] M. Carlson, P. Mucha, and G. Tuck, "Rigid fluid: Animating the interplay between rigid bodies and fluid", ACM Transactions on Graphics(TOG), vol 23, issue 3, August 2004

[4] N. Foster and R. Fedkiw, "Practical animation of liquids", In Proceedings of ACM SIGGRAPH 2001, ACM Press, 23-30. 
[5] T. Hawkins, P. Einarsson, and P. Debevec, "Acquisition of time-varying participating media", ACM Transactions on Graphics(TOG), vol 24, issue 3, July 2005.

[6] G. Irving, E. Guendelman, F. Losasso, and R. Fedkiw, "Efficient simulation of large bodies of water by coupling two and three dimensional techniques", ACM Transactions on Graphics(TOG), vol 25, issue 3, July 2006.

[7] N. Kang, J. Park, J. Noh, and S. Y. Shin, "A Hybrid Approach to Multiple Fluid Simulation using Volume Fractions", Computer Graphics Forum, vol 29, issue 2, 2010

[8] L. Kovar, M. Gleicher, and F. Pighin, "Motion graphs", ACM Transactions on Grpahics(TOG), v.22 n.3, July 2002

[9] J. Krüger and R. Westermann, "Linear algebra operators for GPU implementation of numerical algorithms", $A C M$ Transactions on Graphics (TOG), v.22 n.3, July 2003

[10] J. Lee, J. Chai, P. Reitsma, and J. Hodgins, "Interactive control of avatars animated with human motion data", ACM Transactions on Graphics (TOG), v.21 n.3, July 2002

[11] F. Losasso, F. Gibou, and R. Fedkiw, "Simulating water and smoke with an octree data structure", In $A C M$ SIGGRAPH 2004, 457--462.

[12] Korea Creative Contents Acency, Smart contents market research report,2011

[13] B. Solenthaler and R. Pajarola, "Predictive-corrective incompressible SPH", ACM Transactions on Graphics(TOG), vol 28, issue 3, August 2009.

[14] J. Stam, "Stable fluids", In Proceedings of ACM SIGGRAPH 1999.

[15] M. Vasilescu and D. Terzopoulos, "TensorTextures: multilinear image-based rendering", ACM Transactions on Graphics(TOG), vol 23, issue 3, August 2004

[16] A. Treuille, A. Lewis, and Z. Popovic, "Model reduction for real-time fluids", ACM Transactions on Graphics(TOG), vol 25, issue 3, July 2006

[17] A. Treuille, Y. Lee, and Z. Popovic, "Near-optimal character animation with continuous control", ACM Transactions on Graphics(TOG), vol 26, issue 3, July 2007

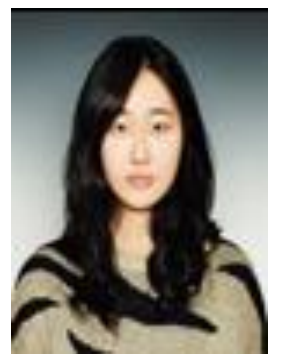

HyangA Ahn is a undergraduate student of Global School of Media, Soongsil University. She is currently working as a undergraduate research assistant of Computational Design Lab at Soongsil University.

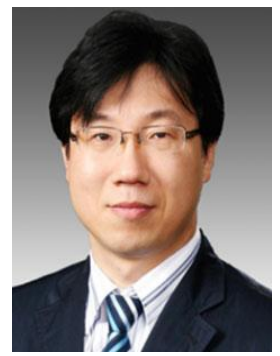

Yong-Ho Seo received his B.S., M.S., and Ph.D. degrees in the Department of Electrical Engineering and Computer Science from KAIST in 1999, 2001, and 2007, respectively. He was an Intern Researcher at the Robotics Group, Microsoft Research, Redmond, WA in 2007. He was a consultant at Qualcomm CDMA Technologies, San Diego, CA, in 2008. Since March 2010, he is currently a Professor and a Chairperson of the Department of Intelligent Robot Engineering, Mokwon University. His research interests include smart device, human-robot interaction, robot vision and wearable computing.

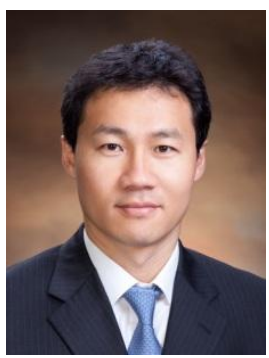

Jinho Park received his B.S. and M.S. degrees in Applied Mathematics in 1999 and 2001, respectively, and Ph.D. in Computer Science in 2007 from Korea Advanced Institute of Science and Technology. He is an assistant professor in the Global School of Media, Soongsil University, South Korea. His research interests include fluid animation and scientific visualization. 